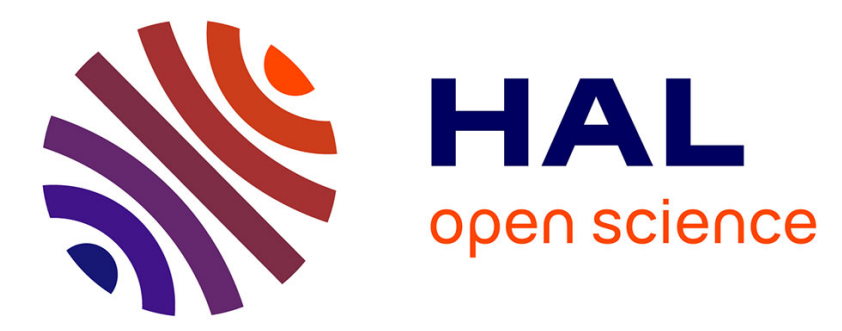

\title{
Nonlinear Brownian dynamics of interfacial fluctuations in a shear flow
}

\author{
Marine Thiébaud, Yacine Amarouchene, Thomas Bickel
}

\section{To cite this version:}

Marine Thiébaud, Yacine Amarouchene, Thomas Bickel. Nonlinear Brownian dynamics of interfacial fluctuations in a shear flow. Journal of Statistical Mechanics: Theory and Experiment, 2014, 2014 (12), pp.P12011 (1-11). 10.1088/1742-5468/2014/12/P12011 . hal-01119909

\author{
HAL Id: hal-01119909 \\ https://hal.science/hal-01119909
}

Submitted on 19 Feb 2016

HAL is a multi-disciplinary open access archive for the deposit and dissemination of scientific research documents, whether they are published or not. The documents may come from teaching and research institutions in France or abroad, or from public or private research centers.
L'archive ouverte pluridisciplinaire HAL, est destinée au dépôt et à la diffusion de documents scientifiques de niveau recherche, publiés ou non, émanant des établissements d'enseignement et de recherche français ou étrangers, des laboratoires publics ou privés.

\section{다)(1) $(5$}

Distributed under a Creative Commons Attribution - NonCommercial| 4.0 International 


\title{
Nonlinear Brownian dynamics of interfacial fluctuations in a shear flow
}

\author{
Marine Thiébaud ${ }^{1}$, Yacine Amarouchene ${ }^{2}$ \\ and Thomas Bickel ${ }^{2}$ \\ ${ }^{1}$ LIPhy, Université de Grenoble and CNRS (UMR 5588), 38402 Saint Martin \\ d'Hères, France \\ ${ }^{2}$ LOMA, Université de Bordeaux and CNRS (UMR 5798), 33405 Talence, \\ France \\ E-mail: yacine.amarouchene@u-bordeaux.fr and thomas.bickel@u-bordeaux.fr
}

\begin{abstract}
Using Brownian dynamics simulations, we investigate the effect of an external flow on the fluctuations of a liquid-liquid interface for a wide range of shear rates. Although the statistics is Gaussian at low shear, we observe a transition to a nonlinear phenomenology above a critical shear rate. In particular, we show that statistical properties at high forcing share striking similarities with Burgers turbulence. An energy criterion allows us to predict the onset of nonGaussian statistics. It also provides a simple explanation for the development of shock singularities through the exchange of kinetic energy from regions with positive to negative gradients in the shear direction.
\end{abstract}

Keywords: turbulence, liquid films (theory), fluids in confined geometries, interfacial phenomena and wetting, soft interfaces 


\section{Contents}

1. Introduction 2

2. Brownian dynamics simulations 4

3. Shock singularities 6

4. Transition from Gaussian to non-linear phenomenology $\quad 7$

5. Discussion 9

$\begin{array}{ll}\text { References } & 10\end{array}$

\section{Introduction}

A fundamental understanding of soft matter systems under shear is a challenging issue [1]. Complexity usually arises from the coupling between the flow and the local structure of the soft material. Despite recent progress on nonequilibrium fluctuation-dissipation relations in sheared systems $[2,3]$, it is never clear a priori whether the injected power should increase [4] or decrease [5] the fluctuations. It is therefore essential to have model systems where theory and experiment can meet in order to capture the relevant features of driven soft materials.

In this article, we investigate the steady-state properties of a liquid-liquid interface in a plane Couette flow at low Reynolds number. Experimentally, interfacial fluctuations can be observed using standard optical microscopy in a phase-separated colloidal dispersion [6] and it was recently shown that thermal capillary waves are strongly reduced by an external shear flow [7]. These observations are in qualitative agreement with subsequent molecular dynamics [8] and Monte-Carlo [9, 10] simulations. An analytical description, which is required in order to be really predictive, was eventually achieved by the present authors within the framework of fluctuating hydrodynamics [11,12]. Still, previous experimental and theoretical studies were limited to relatively low forcing. Our aim is to extend the survey to a wider range of shear rates where nonlinear coupling is prevailing.

This study focuses on the fluctuations of an interface between two immiscible liquids of unequal densities. Properties of the upper (respectively, lower) fluid are labelled with the index $i=1$ (respectively, $i=2$ ). Each fluid is newtonian and incompressible, with viscosity $\eta_{i}$ and mass density $\rho_{i}$. We also define the capillary length $l_{c}=\sqrt{\sigma /\left(\rho_{2}-\rho_{1}\right) g}$ and the capillary time $\tau_{c}=\left(\eta_{1}+\eta_{2}\right) l_{c} / \sigma, g$ being the gravitational acceleration and $\sigma$ the interfacial tension. It is assumed for convenience that the fluctuations are frozen in the direction perpendicular to the shear. We denote $z=h(x, t)$ the local height of the interface with respect to the horizontal $x y$ plane, the projected area being $L \times L$. The two fluids are set in motion along the $x$-direction at constant shear rate $\dot{\gamma}=\left(\eta_{1} \dot{\gamma}_{1}+\eta_{2} \dot{\gamma}_{2}\right) /\left(\eta_{1}+\eta_{2}\right)$, 


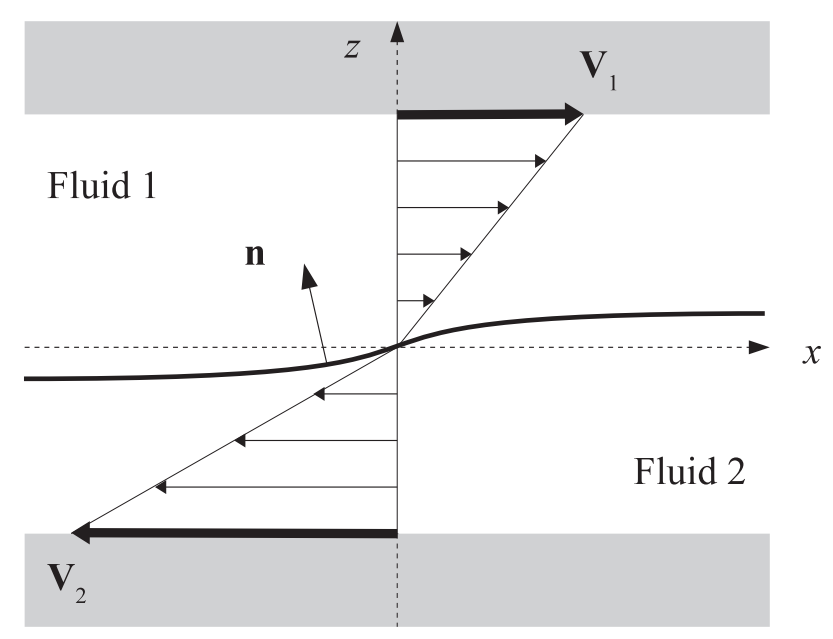

Figure 1. Schematic representation of a liquid interface in a plane Couette flow. The thickness of each fluid layer is $L_{i}$. The fluids are set in motion by moving the boundaries in opposite directions at velocities $V_{i}$.

with $\dot{\gamma}_{i}=V_{i} / L_{i}$-see figure 1 and the discussion in [11]. For convenience, all physical quantities are expressed in dimensionless units: we use $\tau_{c}, l_{c}$ and $k_{\mathrm{B}} T$ respectively as time scale, horizontal length scale (both for $x$ and $q$ ) and energy scale. The position $h$ of the interface is expressed in units of the thermal length $\xi_{T}=\sqrt{k_{\mathrm{B}} T l_{c} /(2 \sigma L)}[6]$. In the limit of vanishing Reynolds number, it was shown recently that the stochastic equation describing the (overdamped) fluctuations of $h(x, t)$ reads, up to second order in $h$ [1]

$$
\partial_{t} h+\frac{\alpha}{\sqrt{2}} h \partial_{x} h=\mathcal{L} h+\varphi(x, t),
$$

with $\varphi$ the white noise and $\mathcal{L} h$ the linear relaxation term. Both terms are conveniently expressed in Fourier representation $h(x, t)=L^{-1} \sum_{q} \tilde{h}_{q}(t) \mathrm{e}^{\mathrm{i} q x}$, with $q=n \times(2 \pi / L)$. The relaxation term is then diagonal in Fourier space: $\mathcal{L} \tilde{h}_{q}=-\lambda_{q} \tilde{h}_{q}$, with $\lambda_{q}=\left(1+q^{2}\right) / 2 q$. The white noise $\tilde{\varphi}_{q}$ follows a Gaussian distribution [11] with $\left\langle\tilde{\varphi}_{q}(t)\right\rangle=0$ and

$$
\left\langle\tilde{\varphi}_{q}(t) \tilde{\varphi}_{q^{\prime}}\left(t^{\prime}\right)\right\rangle=q^{-1} \delta_{q,-q^{\prime}} \delta\left(t-t^{\prime}\right) .
$$

In the absence of shear, equation (1) describes the equilibrium dynamics of overdamped fluctuations [6]. The control parameter that drives the system out of equilibrium is the dimensionless shear rate $\alpha$ defined as

$$
\alpha=\dot{\gamma} \tau_{c} \sqrt{\frac{k_{\mathrm{B}} T}{\sigma L l_{c}}} .
$$

It can be noticed that (1) is a generic equation encountered in various fields, including the KPZ model of surface growth [13] or the Burgers model for turbulence [14]. In the context of phase separation and coarsening under shear, a similar equation has been addressed by the renormalization group [15]. Different dynamics were considered with the conclusion that the linear relaxation term $\mathcal{L} h$ may or may not be relevant at large scales depending on the space dimensionality and the small- $q$ behavior of the eigenvalues $\lambda_{q}$. 

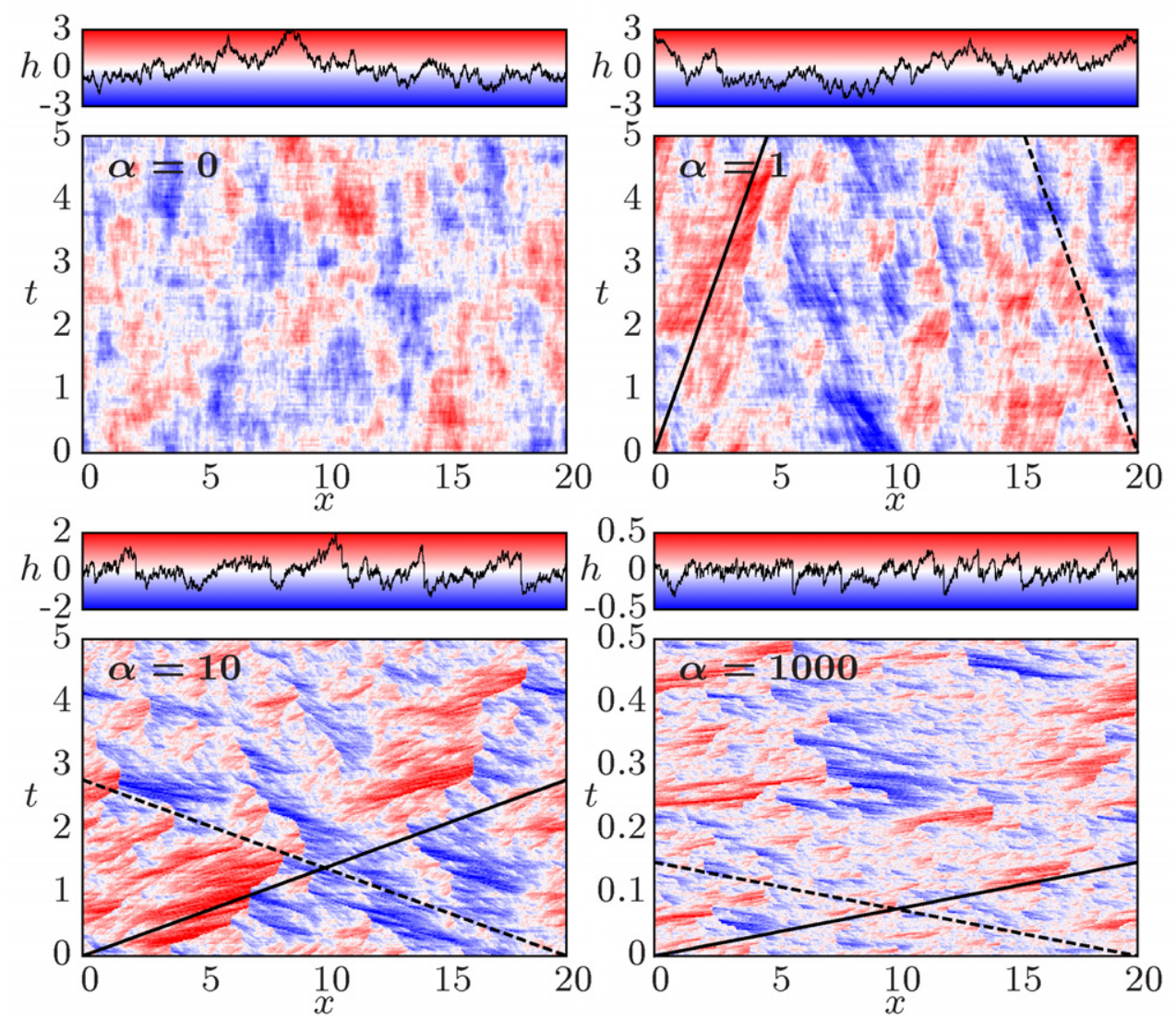

Figure 2. Space-time diagrams of interfacial height whose temporal evolution is given by equation (1). The black lines are guides to the eyes that emphasize for shock propagation.

\section{Brownian dynamics simulations}

The random-forced equation of motion (1) is solved using Brownian dynamics (BD) simulations. We choose a discretization step along the interface $\Delta x=0.002$, the total size of the system being $L=20$ (both in units of $l_{c}$ ). The time step $\Delta t$ is set according to the Courant-Friedrichs-Lewy condition. We also fix the center of mass of the system by setting $\lambda_{0}=0$, in order to remove the nonphysical divergence that may arise when $q \rightarrow 0$. The simulations then proceed from some initial configuration for the interface and evolve forward in time on the basis of (1). The strategy is to go back and forth in direct and reciprocal space in order to handle the different terms. Explicitly, a single time step of the algorithm consists in essentially two parts: (i) Compute the advection according to the equation $\partial_{t} h+\frac{\alpha}{\sqrt{2}} h \partial_{x} h=0$ and then (ii) Fourier transform the result and compute the relaxation and the noise according to $\partial_{t} \tilde{h}_{q}=-\lambda_{q} \tilde{h}_{q}(t)+\tilde{\varphi}_{q}(t)$. The inverse Fourier transform then yields $h(x, t)$ for the next iteration. Typical space-time diagrams are shown in figure 2.

We first discuss the fluctuations at low shear rate $\alpha \ll 1$. In this limit, the variance can be obtained analytically using a perturbative approach [11] and we find

$$
\left\langle h^{2}\right\rangle(\alpha) \approx\left\langle h^{2}\right\rangle_{e q}\left(1-0.028 \alpha^{2}+\ldots\right)
$$



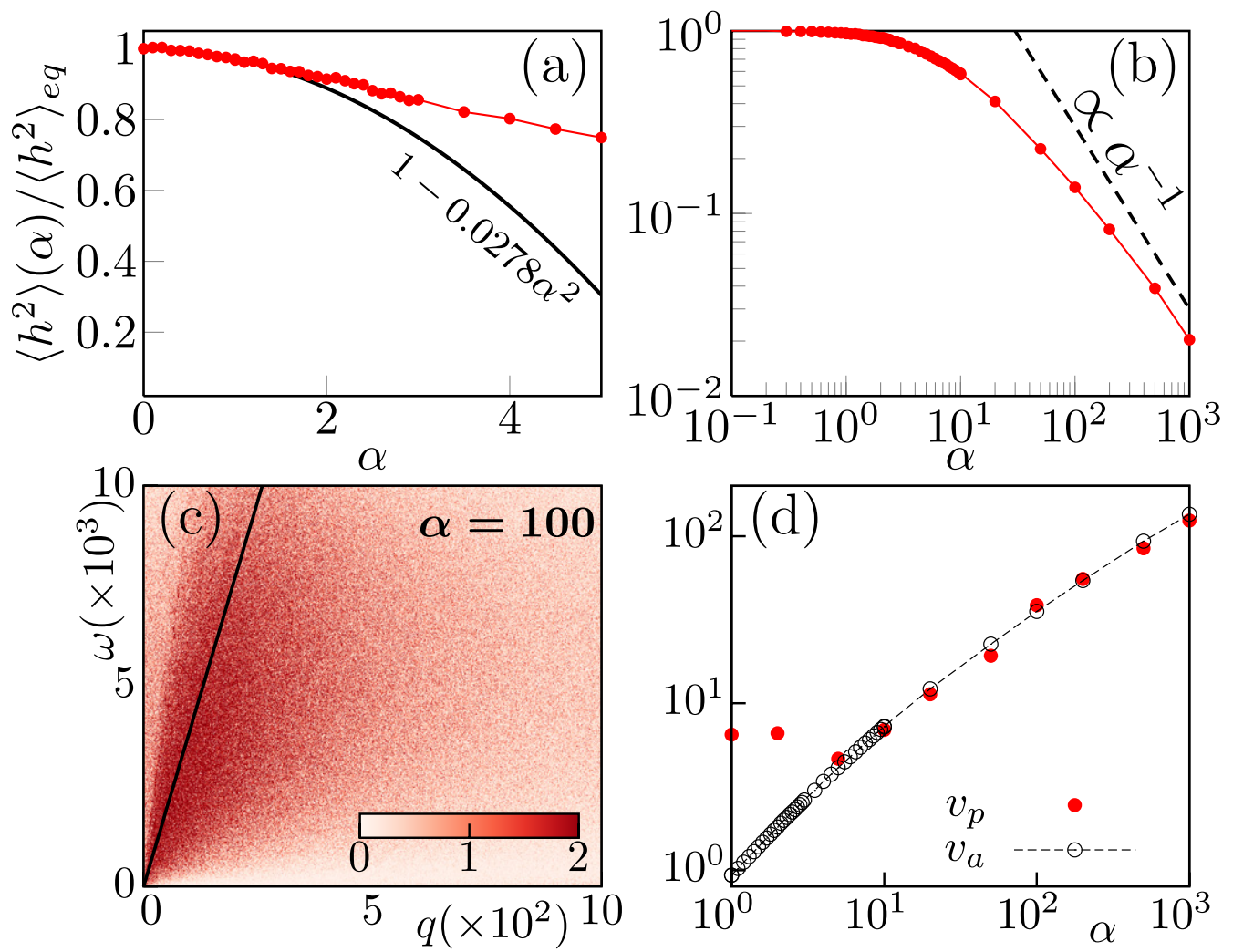

Figure 3. (a) Variance versus shear rate. The solid black line is equation (4). (b) Same as (a) but for larger values of $\alpha$. The dotted line is a guide to the eyes suggesting a power-law decay $\sim \alpha^{-1}$. (c) Spectrum of the interfacial velocity $\partial_{t} h$ in the $(q, \omega)$-plane for $\alpha=100$. The black solid line corresponds to the dispersion relation $\omega=v_{p} q .(d)$ Propagation $\left(v_{p}\right)$ and advection $\left(v_{a}\right)$ velocities versus shear rate.

with $\left\langle h^{2}\right\rangle_{e q}$ the equilibrium variance. As shown in figure $3(a)$, the equilibrium prediction is recovered numercially when $\alpha \rightarrow 0$ and the agreement between simulations and analytical calculations is excellent up to at least $\alpha \approx 1$. These preliminary results thus validate our numerical scheme. In the high shear regime $\alpha>1$, interfacial fluctuations are rapidly flattened by the flow (figure $3(b)$ ). One moreover notices a modification in the morphology of the interface. As can be observed in figure 2, very sharp discontinuities separating smooth regions emerge when the shear rate increases. These discontinuities, or 'shocks', propagate with a characteristic velocity $v_{p}$ that can be extracted from the spectrum of the interfacial velocity $\partial_{t} h$. Indeed, the maximum of the spectrum in the $(q, \omega)$-plane defines a linear dispersion relation $\omega=v_{p} q$-see for instance figure $3(c)^{3}$. One can thus wonder how the propagation velocity $v_{p}$ is related to advection since the shocks are transported by the flow. A characteristic advection velocity $v_{a}$ can be defined from the shear rate and the variance as $v_{a}=\alpha\left\langle h^{2}\right\rangle^{1 / 2}$. figure $3(d)$ reveals that both velocities have the same behavior as the shear rate increases, proving that the characteristic advection velocity coincides with the velocity of shock propagation.

3 Note that, in the absence of shear, there is no propagation since deformation modes are overdamped. 


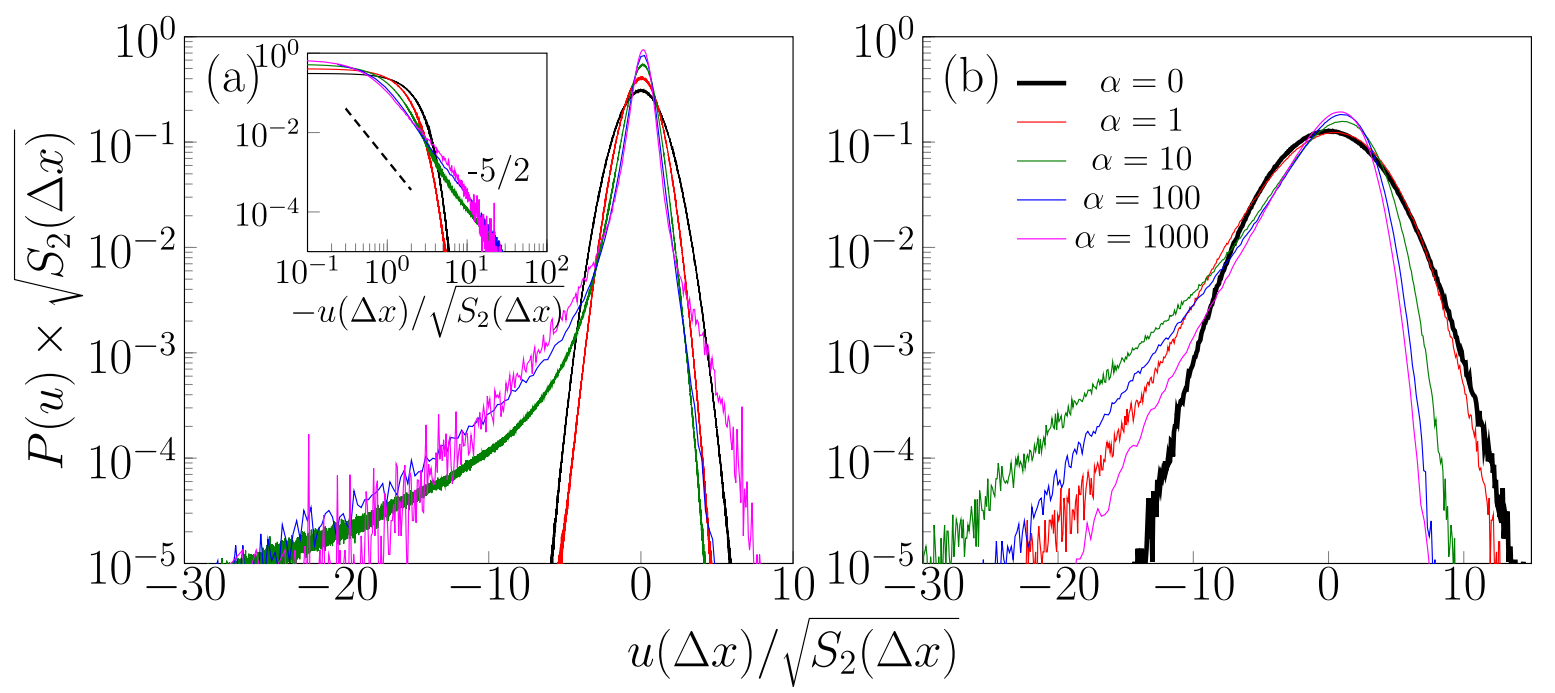

Figure 4. $\mathrm{PDF}$ of the increments $u(\Delta x)=h(x+\Delta x)-h(x)$ with $\Delta x=0.004 l_{c}(a)$ and $\Delta x=0.1 l_{c}(b)$. Both $u$ and $P(u)$ are normalized by the variance $S_{2}(\Delta x)^{1 / 2}$ (see text). The color code defined in $(b)$ is shared by both figures. Inset of $(a)$ : $\log -\log$ plot of the left tail of the distribution. The dotted line is a guide to the eyes suggesting a power-law with an exponent $-5 / 2$.

\section{Shock singularities}

The singularities of the interface profile are reminiscent of the shocks observed in the context of Burgers turbulence [14,16]. The Burgers equation is a simplified 1D version of the Navier-Stokes equation; it provides a useful benchmark to test theoretical issues such as scaling and multifractality in turbulence. The random-force-driven Burgers equation is in every respect similar to the equation (1), excepted that the linear relaxation term reads $\mathcal{L} h=\nu \partial_{x x} h$, with $\nu$ the kinematic viscosity ${ }^{4}$. It was shown that the distribution of velocity gradients is of particular interest since it is characterized by anomalous scaling exponents [17-20]. Here we consider the statistical properties of the height gradients, or increments, that we define as $u(\Delta x)=h(x+\Delta x)-h(x)$. A typical set of probability distribution functions (PDF) for the random variable $u$ is shown on figure 4 . The distribution is Gaussian at equilibrium $(\alpha=0)$, but becomes asymmetric as soon as the flow is switched on. Indeed, regions with positive slopes are flattened by the flow although regions with negative slopes are increasingly favorable - eventually leading shock singularities. The rare fluctuations (corresponding to the left tails of the distributions) are strongly nonGaussian and seem to lead to a power law at small scales (see inset of figure 4(a)). The situation differs at larger scales since rare events seem to be exponentially distributed (see figure $4(b)$ ), presumably due to the averaging over several shocks.

To get further insight, we focus on the moments of the increment distribution defined by $S_{p}=\left\langle|u(\Delta x)|^{p}\right\rangle$. Following an analysis that is common in the field of turbulence [14],

\footnotetext{
4 In the context of the Burgers equation, the viscosity $\nu$ is only taken into account in order to ensure numerical
} stability. The statistical properties are then obtained in the limit $\nu \rightarrow 0$. 

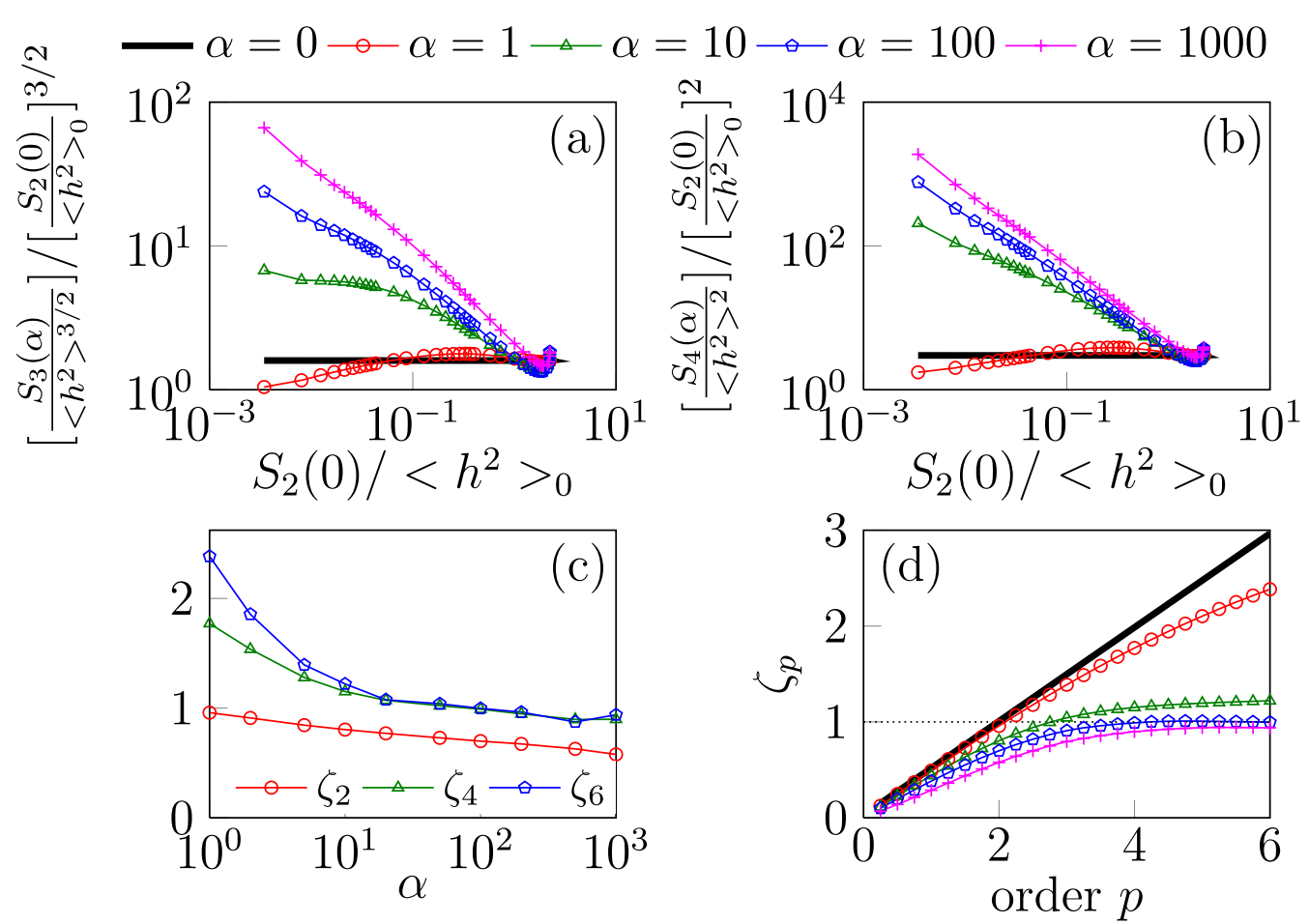

Figure 5. Normalized structure functions $S_{p}$ as a function of the increment $\Delta x$, for $p=3(a)$ and $4(b)$. (c) Scaling exponents $\zeta_{2}, \zeta_{4}$ and $\zeta_{6}$ versus $\alpha$. $(d)$ Scaling exponents as a function of the order $p$ of the moment. The full black line corresponds to the equilibrium law $\zeta_{p}(0)=p / 2$.

one can expect the moments to scale as

$$
S_{p} \sim \Delta x^{\zeta_{p}}
$$

with $\zeta_{p}$ a scaling exponent. Note that the order $p$ of the moment does not need to be an integer. We first check that, at equilibrium, the second moment scales as $S_{2}(\alpha=0) \propto \Delta x$ when $\Delta x \ll 1$. We then plot higher moments normalized by $S_{2}(\alpha=0)^{p / 2}$ as function of $S_{2}(\alpha=0)$, in accordance to the extended self-similarity method [21] - see for instance figures $5(a)$ and $(b)$. The scaling exponents $\zeta_{p}(\alpha)$ are then extracted by fitting the curves (see figure $5(c)$ ) and the results are summarized in figure $5(d)$ that shows the evolution of $\zeta_{p}$ as a function of the order $p$. At thermal equilibrium $(\alpha=0)$ the PDF is Gaussian and we find $\zeta_{p}(0)=p / 2$, as expected for Brownian fluctuations. The scaling exponents $\zeta_{p}$ (with $p \geqslant 3$ ) then strongly deviate from the $p / 2$ law as the shear rate increases and eventually saturate to a value close to unity when $\alpha \gg 1$. This saturation is also observed theoretically for Burgers turbulence, where it is interpreted in terms of bifractality [14] and experimentally for a deformable material line in 2D turbulence [22].

\section{Transition from Gaussian to non-linear phenomenology}

The analysis of increments reveals a cross-over from a Gaussian to a nonlinear behavior, the transition being triggered by the shear rate. Two questions then naturally arise: (i) is 


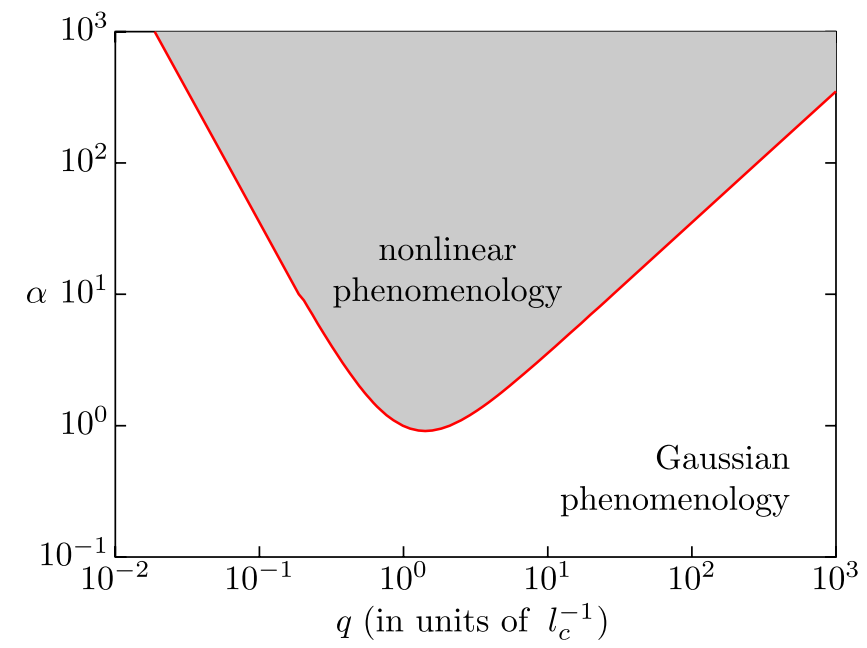

Figure 6. 'Phase diagram' describing the transition from Gaussian to nonlinear dynamics.

there a critical shear rate above which the phenomenology switches from Gaussian to nonlinear and (ii) are all the spatial scales affected in the same way? To answer these important issues, let us consider the kinetic energy density $e_{c}$ of the interface. The latter can be defined from the interfacial relaxation velocity $u=\partial_{t} h$ as $e_{c}=u^{2} / 2$. From (1) one immediately gets the following time evolution equation

$$
\partial_{t} e_{c}+\frac{\alpha}{\sqrt{2}} \partial_{x}\left(h e_{c}\right)=u \mathcal{L} u-\frac{\alpha}{\sqrt{2}} e_{c} \partial_{x} h+\phi,
$$

with $\phi=u \partial_{t} \varphi$ the noise term. Besides the usual contributions from advection $\left(\partial_{x} h e_{c}\right)$ and relaxation $(u \mathcal{L} u)$, there is an additional forcing term which is proportional to the local slope $\partial_{x} h$. If the slope is negative (resp. positive), this contribution plays the role of a source (resp. sink) term. As such, it accounts for the exchange of kinetic energy from regions with positive slopes towards regions with negative slopes. This term thus outlines how the nonlinearities actually modify the spectral properties of the interface.

The competition between nonlinear forcing and relaxation can be quantified by the dimensionless number $M$

$$
M=\frac{\alpha}{\sqrt{2}}\left|\frac{e_{c} \partial_{x} h}{u \mathcal{L} u}\right| .
$$

If $M<1$ the phenomenology is essentially Gaussian, whereas nonlinear effects dominate when $M>1$. In order to understand how spatial scales are affected, we consider a single deformation mode $h(x, t)=h_{q} \mathrm{e}^{\mathrm{i}(q x-\omega t)}$. The number $M_{q}$ at a given wavelength $q$ can be estimated if one assumes that $h_{q}$ follows an initial equilibrium distribution with $\left\langle\left|h_{q}\right|^{2}\right\rangle \sim 1 /\left(1+q^{2}\right)$. We then find that $M_{q} \sim \alpha F(q)$ with $F(q)=q^{2} /\left(1+q^{2}\right)^{3 / 2}$. The function $F(q)$ is bounded: $0 \leqslant F(q) \leqslant F_{\max }$, the extremal value $F_{\max } \sim \mathcal{O}(1)$ being reached for $q_{\max } \sim \mathcal{O}(1)$. As a consequence, we predict that there exists a critical shear rate $\alpha_{c} \sim \mathcal{O}(1)$ below which the dynamics is purely Gaussian. Above $\alpha_{c}$, there is an increasing range of scales $q_{-}<q<q_{+}$that are affected by nonlinear effects - see figure 6 . Note also that larger scales are affected before the smaller ones at high shear since $q_{-} \sim \alpha^{-1 / 2}$ and $q_{+} \sim \alpha$ in the limit $\alpha \gg 1$. 

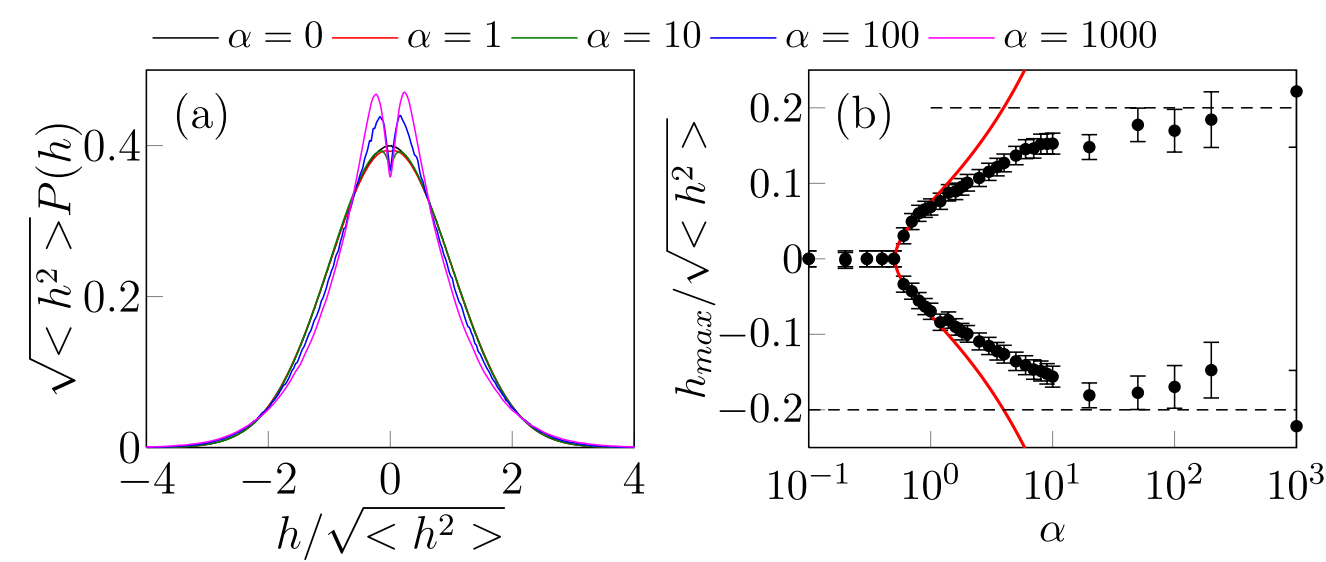

Figure 7. (a) PDF of the vertical displacement $P(h)$ versus shear rate. (b) Values of the maxima of the PDF versus shear rate. The full red line corresponds to equation (8).

As an illustration of this scenario, we compute the probability distribution functions $P(h)$ for the local height $h$ of the interface. The PDF are shown in figure 7( $a)$ for different values of $\alpha$. The distributions are Gaussian at low shear but split into two symmetric parts above a certain threshold. The most probable position then switches from $h=0$ to $h= \pm h_{\max }$ as shown in figure $7(b)$. Close to the threshold $\alpha_{c} \approx 0.51$, the bifurcation is characterized by a power-law $\left(\alpha>\alpha_{c}\right)$

$$
h_{\max }=A\left(\alpha-\alpha_{c}\right)^{\beta} \text {, }
$$

with $A \approx 0.10$ and $\beta \approx 0.50$. At higher shear rates $\alpha \gg \alpha_{c},\left|h_{\max }\right|$ seems to saturate to $\left|h_{\max }\right| \approx 0.2\left\langle h^{2}\right\rangle_{e q}$. Note that the bifurcation is expected to be difficult to observe using confocal microscopy since it would occur below the diffraction limit $\left(\left\langle h^{2}\right\rangle_{e q} \approx 1 \mu \mathrm{m}\right.$ in the experiment of Derks et al [7]). Still, other techniques such as light scattering [23] are much more efficient to detect thermally excited surface waves and could thus be used to highlight this scenario of symmetry breaking.

\section{Discussion}

To summarize, we have analyzed the statistical properties of an interface in a Couette flow for a wide range of shear rates. Our study reveals strong similarities with Burgers turbulence. This is especially striking since the relaxation equation (1) was derived for overdamped fluctuations in the limit of vanishing Reynolds number, whereas turbulence and the Burgers equation are studied in the limit of high Reynolds number. As a matter of fact, a renormalization group ( $R G$ ) analysis carried out recently [15] has shown that the relevance of the different terms in (1) depends essentially on the spatial dimensionality $d$ and on the asymptotic behavior of $\lambda_{q}$. Here, we have $\lambda_{q} \sim q$ (for length scales below the capillary length), so that the critical dimension is $d_{c}=3$ [15]. Since $d=2$ in this work, the linear relaxation term flows to zero in $\mathrm{RG}$ recursion relations so that the linear contribution is actually irrelevant. The $\mathrm{RG}$ analysis thus provides a plausible explanation to the fact that the two models, although appropriate in opposite limits (small versus 
large Reynolds number), exhibit similar statistical properties. Note that this conclusion is specific to $d=2$ and might not hold for higher spatial dimensionality (e.g. $d=3$ ).

Still, the derivation of the time-evolution equation (1) is based on several simplifying assumptions [11]. First, only the lowest-order nonlinearity is retained, namely, the advection of height deformation by the flow. In principle, higher-order terms can be determined as well (even if the calculations might become quite cumbersome), but they are expected to be less and less relevant as the shear rate increases (at least as far as the second moment is concerned). The second point is that equation (1) is derived in the limit of vanishing Reynolds number, but it is known that inertial effects can be destabilizing at small but finite Reynolds number (see [12] and references therein). It would thus be interesting to study the competition between both nonlinearities, arising respectively from advection and fluid inertia. Thirdly, it is implicitly assumed in (1) that the thickness of both fluid layers is much larger than the capillary length: $L_{1}, L_{2} \gg l_{c}$. It was recently shown in the opposite limit $L_{1}, L_{2} \ll l_{c}$ that the coupling between flow and interfacial fluctuations can have major repercussions on the dynamics of wetting [24]. Taking confinement into account would therefore be another interesting opening.

In conclusion, the main predictions of this work regard the phenomenology transition from Gaussian to nonlinear as the shear rate increases. Below a critical shear rate $\alpha_{c} \sim \mathcal{O}(1)$, the statistics show only slight deviations from Gaussian: this justifies a posteriori the perturbative calculations of [11]. On the other hand, above $\alpha_{c}$, the shear affects in a nonlinear manner the modes with wavelengths comparable to the capillary length, in agreement with the handwaving argument presented in [7]. Finally, one can note that the nonlinear phenomenology, usually referred as the Burgers phenomenology, can extend far below the high Reynolds number regime. Our findings might thus shed new light on driven soft matter systems, ranging from shock waves in 2D microfluidics $[25,26]$ to shock-wave formation [27] or capillary-like phenomena [28] in granular flows.

\section{References}

[1] Cates M E and Evans M R (ed) 2000 Soft and Fragile Matter: Nonequilibrium Dynamics, Metastability and Flow (Bristol: Institute of Physics)

[2] Baule A and Evans R M L 2008 Phys. Rev. Lett. 101240601

[3] Speck T and Seifert U 2009 Phys. Rev. E 79040102

[4] Ortiz de Zárate J M and Sengers J V 2009 Phys. Rev. E 79046308

[5] Bruinsma R and Rabin Y 1992 Phys. Rev. A 45994

[6] Aarts D G A L, Schmidt M and Lekkerkerker H N W 2004 Science 304847

[7] Derks D, Aarts D G A L, Bonn D, Lekkerkerker H N W and Imhof A 2006 Phys. Rev. Lett. 97038301

[8] Thakre A K, Padding J T, den Otter W K and Briels W J 2008 J. Chem. Phys. 129044701

[9] Smith T H R, Vasilyev O, Abraham D B, Maciolek A and Schmidt M 2008 Phys. Rev. Lett. 101067203

[10] Smith T H R, Vasilyev O, Maciolek A and Schmidt M 2010 Phys. Rev. E 82021126

[11] Thiébaud M and Bickel T 2010 Phys. Rev. E 81031602

[12] Thiébaud M and Bickel T 2011 Soft Matter 77813

[13] Kardar M, Parisi G and Zhang Y C 1986 Phys. Rev. Lett. 56889

[14] Bec J and Khanin K 2007 Phys. Rep. 4471

[15] Bray A J, Cavagna A and Travasso R D M 2001 Phys. Rev. E 65016104

[16] Frisch U 1995 Turbulence: the Legacy of A N Kolmogorov (Cambridge: Cambridge University Press)

[17] Chekhlov A and Yakhot V 1995 Phys. Rev. E 525681

[18] Hayot F and Jayaprakash C 1997 Phys. Rev. E 564259

[19] Bec J 2001 Phys. Rev. Lett. 87104501 
[20] Mitra D, Bec J, Pandit R and Frisch U 2005 Phys. Rev. Lett. 94194501

[21] Arnéodo A et al 1996 Europhys. Lett. 34411

[22] Amarouchene Y and Kellay H 2005 Phys. Rev. Lett. 95054501

[23] Raudsepp A, Fretigny C, Lequeux F and Talini L 2012 Rev. Sci. Instrum. 83013111

[24] Fetzer, R, Rauscher M, Seemann R, Jacobs K and Mecke K 2007 Phys. Rev. Lett. 99114503

[25] Beatus T, Tlusty T and Bar-Ziv R 2009 Phys. Rev. Lett. 103114502

[26] Beatus T, Bar-Ziv R and Tlusty T 2012 Phys. Rep. 516103

[27] Amarouchene Y and Kellay H 2006 Phys. Fluids 18031707

[28] Amarouchene Y, Boudet J F and Kellay H 2008 Phys. Rev. Lett. 100218001 